Reprod. Nutr. Dévelop., 1981, 21 (3), 429-440.

\title{
Crise audiogène chez la souris selon la souche et le sexe : influence de la ration magnésique et des neuromédiateurs
}

par P. BAC

\author{
Laboratoire de Biologie animale, \\ Faculté des Sciences pharmaceutiques ef biologiques, \\ rue Jean-Baptiste Clément, 92290 Châtenay Malabry, France.
}

Summary. The influence of strain, sex, magnesium deficiency and brain biogenic amines on audiogenic seizures in mice.

Mice of the DBA/2/M strain are audio-sensitive only for a short period (5 to 10 days). When this period is over, 100 p. 100 audiogenic seizures can be obtained with experimental magnesium deficiency. When $A K R / R, O F 1$ and $C 57 B L / R$ mice were deprived of magnesium for 15 or 21 days, they manifested an audiogenic attack only when given amphetamine sulfate, caffeine or isonicotinylhydrazine. If they had been deprived for 43 days, it was no longer necessary to administer these substances. The audiogenic attack could be prevented by correcting the deficiency with magnesium chloride or by preliminary administration of a single dose of various barbiturics, anti-convulsives, tranquillizers or parachlorophenylalanine, but not neuroleptics.

\section{Introduction.}

A la suite de l'observation de la crise audiogène chez la souris par Stoudenson en 1924 et chez le rat par Donaldson, également en 1924, de très nombreux auteurs ont entrepris l'étude de cette crise sur le plan génétique et neuropharmacologique (Fuller et Sjursen, 1967 ; Boggan ef al., 1971 ; Deckard et al., 1976). Il est connu que la carence magnésique introduit un étał d'hypersensibilité neuromusculaire (Griffiths, 1947 ; Suter ef al., 1957 ; Chutkow, 1965 ; Buck, Mahoney ef Hendricks, 1976) chez l'animal ef chez l'homme (Durlach, 1976, 1977). Toutefois assez peu de travaux ont été effectués, selon le sexe, sur des souris carencées en magnésium appartenant à différentes lignées. Les effets des convulsivants comme l'isoniazide et la caféine étudiés chez l'animal normal (Buchel et al., 1961 ; Busnel et Lehmann, 1961 ; Chauchard ef Mazoue, 1963 ; Lehmann, 1963) ne l'ont pas été chez l'animal carencé en magnésium.

Il est établi que les barbituriques, les tranquillisants ef les anti-convulsivants sont capables de protéger les souris de lignées « sensibles» contre la crise audiogène (Simler et al., 1968 ; 1971 ; Cielsinsky, Maitre et Simler, 1972) mais, à notre connais- 
sance, aucun travail n'a été effectué dans ce sens chez la souris carencée en magnésium.

De même il est reconnu que l'amphétamine exerce une action protectrice contre la crise audiogène (Lehmann, 1967). A la suite d'expériences portant sur les réactions nociceptives chez la souris nous avons constaté que cette substance agit de façon différente selon qu'il s'agit d'un animal normal ou carencé en magnésium et nous avons voulu préciser son action.

Le but de cette étude est de :

1) déterminer la durée de carence magnésique nécessaire pour que des souris de lignées différentes (OF1, C57BL/R, AKR/R, DBA/2/M) soient sensibles à la crise audiogène et de rechercher si la simple correction de la carence par le chlorure de magnésium permet de supprimer cette crise :

2) savoir si différentes substances (barbituriques, amphétamine, caféine, isoniazide, parachlorophénylalanine, neuroleptiques et anticonvulsivants) qui agissent sur les neuromédiateurs exercent une action sur la crise audiogène de la souris carencée en magnésium.

\section{Matériel et méthodes.}

Le régime carencé en magnésium a été préparé au laboratoire et correspondait à celui de Voskian (1970). Il contenait $10 \mathrm{mg}$ de magnésium par $\mathrm{kg}$. L'aliment témoin en contenait $1 \mathrm{~g}$ (sous forme de chlorure de magnésium) ce qui suffisait aux besoins quotidiens des animaux.

Dans cette éfude nous avons utilisé 4 souches de souris IOPS *. La première était une souche non consanguine (OF1) et les 3 autres l'étaient (AKR/R, C57BL/R, DBA/2/M). Nous savons que la sensibilité à la crise audiogène diminue en fonction de l'âge (Ginsburg, 1963 ; Swinyard, 1963) ; le tableau 1 résume, pour chaque souche, la sensibilité

TABLEAU 1

Sensibilité à la crise audiogène

\begin{tabular}{|c|c|c|c|c|c|c|}
\hline Souches & Consanguines & Non consanguines & $\dot{\mathrm{a}} \mathrm{I}$ & $\begin{array}{l}\text { Sensit } \\
\text { crise }\end{array}$ & $\begin{array}{l}\text { ilité } \\
\text { zudiogèn }\end{array}$ & \\
\hline âge $\ldots \ldots \ldots$ & & & $\begin{array}{l}35 j \\
\text { p. } 100\end{array}$ & $\begin{array}{c}55 \mathrm{j} \\
\text { p. } 100\end{array}$ & $\begin{array}{c}70 \mathbf{j} \\
\text { p. } 100\end{array}$ & sexe \\
\hline OF1 $\ldots \ldots \ldots$ & & + & 0 & 0 & 0 & $\hat{0}$ \\
\hline AKR/R $\ldots \ldots$ & + & & 0 & 0 & 0 & 0 \\
\hline C57BL/R ..... & & + & 0 & 0 & 0 & $\hat{0}$ \\
\hline $\mathrm{DBA} / 2 / \mathrm{M} \ldots \ldots$ & & + & 95 & 5 & 0 & 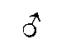 \\
\hline
\end{tabular}

* IOPS : Indemnes d’Organismes Pathogènes Spécifiques. 
à la crise audiogène. Afin de démontrer le rôle sensibilisant de la carence magnésique dans cette crise nous avons utilisé des animaux adultes, capables de se reproduire, et nous avons vérifié sur des lots témoins, non carencés en magnésium, que les animaux des souches précédentes, dans nos conditions expérimentales, étaient insensibles à la crise audiogène.

Cette crise a été déclenchée par l'émission, dans une enceinte de $50 \times 60 \times 20 \mathrm{~cm}$, d'un son de fréquence $10 \mathrm{kHz} \pm 100 \mathrm{~Hz}$. La puissance sonore a été fixée, du fait de la grande sensibilité des animaux carencés (Chutkow, 1971), à $80 \mathrm{~dB}( \pm 0,3 \mathrm{~dB})$ pour l'étude de la crise audiogène chez diverses souches de souris, et à $120 \mathrm{~dB}( \pm 0,3 \mathrm{~dB})$ pour l'étude des substances protectrices de la crise audiogène. Lors du test audiogène une seule souris était présente dans l'enceinte. Nous avons vérifié que la crise audiogène chez la souris carencée en magnésium était identique à celle des animaux de lignées « sensibles 》 (Frings et al., 1952). Au cours de cette étude nous n'avons tenu compte que des crises complètes (course, phase clonique, phase tonique).

\section{Résultats.}

1. Crise audiogène ef ration magnésique.

Crise audiogène en fonction de la durée de la carence. - Les animaux étaient répartis en $\mathbf{8}$ groupes selon la souche et le sexe. L'âge ef le poids sont indiqués au tableau 2. Dans chaque groupe les divers lots correspondaient à un temps de carence déterminé. Les résultats sont regroupés dans le tableau 3. On retiendra que le chiffre supérieur figurant dans chaque case de ce tableau indique le nombre de crises audiogènes et le chiffre inférieur le nombre d'animaux utilisés.

Pour la souche OF1, le nombre de crises audiogènes a été très faible jusqu'au $27 \mathrm{e}$ jour de carence, puis il est devenu très important à partir du $33^{\mathrm{e}}$ jour. Pour les femelles de la souche C57BL/R la sensibilité est apparue dès le $21^{\mathrm{e}}$ jour. Pour celles de la souche $A K R / R$ la sensibilité est apparue un peu plus tôt, alors que pour les mâles elle est apparue à partir du $21^{\mathrm{e}}$ jour. Les souris de la souche DBA/2/M étaient presque toutes sensibles au $19 \mathrm{e}$ jour, ef en totalité à partir du $21 \mathrm{e}$ jour.

Correction de la crise audiogène par le chlorure de magnésium (łabl. 4). - Sur des lots et des souches identiques aux précédents, le chlorure de magnésium a été administré en solution dans l'eau distillée, par sonde œsophagienne à la dose de $14 \mathrm{mg}$ $\left(\mathrm{MgCl}_{2}, 6 \mathrm{H}_{2} \mathrm{O}\right)$ par souris et par $24 \mathrm{~h}$ pendant une durée de 10 jours. Après un tel traitement tous les animaux étaient insensibles à la crise audiogène (tabl. 4).

2. Substances protectrices de la crise audiogène.

Action des tranquillisants, des barbituriques ef des anti-convulsivants (tabl. 5). - Pour cet essai nous n'avons utilisé que des souris OF1 réparties en 2 groupes selon le sexe. Les animaux, du seul fait de la durée de leur carence ( 43 jours), étaient sensibles à la crise audiogène déclenchée par l'émission d'une fréquence de $10 \mathrm{kHz}$ et d'intensité $120 \mathrm{~dB}$. Toutes les substances ont été administrées en intrapéritonéale $30 \mathrm{~min}$ avant le test audiogène. 
TABLEAU 2

Poids des souris en fonction de la durée de la carence

\begin{tabular}{|c|c|c|c|c|c|c|c|c|c|}
\hline \multirow{3}{*}{$\underset{\text { (j) }}{\text { Age }}$} & \multirow{3}{*}{$\begin{array}{l}\text { Durée de } \\
\text { carence } \\
\text { (j) }\end{array}$} & \multicolumn{8}{|c|}{ Poids (g) } \\
\hline & & \multicolumn{2}{|c|}{ OF1 } & \multicolumn{2}{|c|}{$\mathrm{C} 57 \mathrm{BL} / \mathrm{R}$} & \multicolumn{2}{|c|}{$A K R / R$} & \multicolumn{2}{|c|}{$\mathrm{DBA} / 2 / \mathrm{M}$} \\
\hline & & $\hat{0}$ & 우 & $\hat{0}$ & 우 & $\delta$ & 우 & $\hat{0}$ & $q$ \\
\hline 70 & 0 & $\begin{array}{l}26 \\
\pm \\
0,3\end{array}$ & $\begin{array}{l}23 \\
\pm \\
0,4\end{array}$ & $\begin{array}{l}22 \\
\pm \\
0,3\end{array}$ & $\begin{array}{c}19,3 \\
\pm \\
0,4\end{array}$ & $\begin{array}{l}23 \\
\pm \\
0,2\end{array}$ & $\begin{array}{l}22 \\
\frac{\overline{0}}{0,3}\end{array}$ & $\begin{array}{l}22 \\
\pm \\
0,3\end{array}$ & $\begin{array}{l}19 \\
\pm \\
0,2\end{array}$ \\
\hline 80 & 10 & $\begin{array}{c}27,2 \\
\pm \\
0,6\end{array}$ & $\begin{array}{c}24,1 \\
\pm \\
0,3\end{array}$ & $\begin{array}{c}22,4 \\
\pm \\
0,8\end{array}$ & $\begin{array}{c}20,2 \\
\pm \\
0,4\end{array}$ & $\begin{array}{c}24,3 \\
\pm \\
0,5\end{array}$ & $\begin{array}{c}22,8 \\
\pm \\
0,6\end{array}$ & $\begin{array}{c}23,8 \\
\pm \\
0,2\end{array}$ & $\begin{array}{c}20,2 \\
\pm \\
0,4\end{array}$ \\
\hline 85 & 15 & $\begin{array}{c}27,4 \\
\pm \\
0,3\end{array}$ & $\begin{array}{c}24,6 \\
\pm \\
0,3\end{array}$ & $\begin{array}{c}22,8 \\
\pm \\
0,2\end{array}$ & $\begin{array}{c}20,8 \\
\pm \\
0.4\end{array}$ & $\begin{array}{c}25,1 \\
\pm \\
0,2\end{array}$ & $\begin{array}{l}23 \\
\pm \\
0,4\end{array}$ & $\begin{array}{c}24,2 \\
\pm \\
0,3\end{array}$ & $\begin{array}{c}21,6 \\
\pm \\
0,2\end{array}$ \\
\hline 89 & 19 & $\begin{array}{c}27,9 \\
\pm \\
0,6\end{array}$ & $\begin{array}{c}25,8 \\
\pm \\
0,8\end{array}$ & $\begin{array}{c}23 \\
\pm \\
0,4\end{array}$ & $\begin{array}{c}21,4 \\
\pm \\
0,3\end{array}$ & $\begin{array}{c}25,8 \\
\pm \\
0,2\end{array}$ & $\begin{array}{c}23,4 \\
\pm \\
0,5\end{array}$ & $\begin{array}{l}25,2 \\
\pm \\
0,4\end{array}$ & $\begin{array}{c}21,8 \\
\pm \\
0,3\end{array}$ \\
\hline 91 & 21 & $\begin{array}{c}28,2 \\
\pm \\
0,6\end{array}$ & $\begin{array}{c}26,2 \\
\pm \\
0,2\end{array}$ & $\begin{array}{c}25 \\
\pm \\
0,3\end{array}$ & $\begin{array}{c}22,8 \\
\pm \\
\frac{ \pm}{0,6}\end{array}$ & $\begin{array}{c}26 \\
\frac{ \pm}{ \pm} \\
0,2\end{array}$ & $\begin{array}{c}24,2 \\
\pm \\
0,6\end{array}$ & $\begin{array}{l}26 \\
\pm \\
0 ; 2\end{array}$ & $\begin{array}{c}22,4 \\
\pm \\
0,6\end{array}$ \\
\hline 97 & 27 & $\begin{array}{l}29 \\
\pm \\
0,3\end{array}$ & $\begin{array}{c}27,4 \\
\pm \\
\pm 0,4\end{array}$ & $\begin{array}{c}27,6 \\
\pm \\
0,8\end{array}$ & $\begin{array}{c}25,4 \\
\pm \\
0,6\end{array}$ & $\begin{array}{c}27,2 \\
\pm \\
0,6\end{array}$ & $\begin{array}{c}26,4 \\
\pm .3 \\
0,3\end{array}$ & & \\
\hline 103 & 33 & $\begin{array}{c}29,6 \\
\pm \\
0,3\end{array}$ & $\begin{array}{c}28,2 \\
\pm \\
0,8\end{array}$ & $\begin{array}{c}28,4 \\
\pm \\
0,6\end{array}$ & $\begin{array}{c}26,3 \\
\pm \\
0,2\end{array}$ & $\begin{array}{c}29,6 \\
\pm \\
0,4\end{array}$ & $\begin{array}{c}27,6 \\
\pm \\
0,2\end{array}$ & & \\
\hline 113 & 43 & $\begin{array}{c}30,8 \\
\pm \\
0,8\end{array}$ & $\begin{array}{c}29,4 \\
\pm \\
0,6\end{array}$ & $\begin{array}{c}28,4 \\
\pm \\
0,2\end{array}$ & $\begin{array}{l}26,9 \\
\pm \\
0,4\end{array}$ & $\begin{array}{c}29,8 \\
\pm \\
0,3\end{array}$ & $\begin{array}{c}28,4 \\
\pm \\
0,6\end{array}$ & & \\
\hline
\end{tabular}

Le méprobamate, le phénobarbital, l'amobarbital, le diazépam et le dipropylacétate de sodium ont empêché l'apparition des crises audiogènes chez tous les animaux ainsi traités. Ceci confirme, pour l'animal carencé en magnésium, les travaux de Mercier (1953) portant sur l'animal « sensible » à la crise audiogène.

Action de la parachlorophénylalanine (PCPA) (tabl. 6). - Pour cet essai nous n'avons utilisé que des souris mâles appartenant aux 4 souches précédemment étudiées. La PCPA à la dose de $300 \mathrm{mg} / \mathrm{kg}$ a été administrée en intrapéritonéale $24 \mathrm{~h}$ avant la stimulation sonore; elle protège totalement les souris de souche OF1 et $C 57 \mathrm{BL} / \mathrm{R}$ ef partiellement celles de souches $A K R / R$ et $D B A / 2 / M$. 
TABLEAU 3

Crise audiogène en fonction de la durée de carence

\begin{tabular}{|c|c|c|c|c|c|c|c|c|}
\hline \multirow{2}{*}{$\begin{array}{c}\text { Durée de la } \\
\text { carence (j) }\end{array}$} & \multicolumn{2}{|c|}{ OF1 } & \multicolumn{2}{|c|}{$\mathrm{C} 57 \mathrm{BL} / \mathrm{R}$} & \multicolumn{2}{|c|}{$A K R / R$} & \multicolumn{2}{|c|}{$\mathrm{DBA} / 2 / \mathrm{M}$} \\
\hline & 0 & q & $\hat{0}$ & q & 0 & ㅇ & $\hat{\sigma}$ & q \\
\hline 0 & $\begin{array}{r}0 \\
20\end{array}$ & $\begin{array}{r}0 \\
20\end{array}$ & $\begin{array}{r}0 \\
20\end{array}$ & $\begin{array}{r}0 \\
20\end{array}$ & $\begin{array}{r}0 \\
24\end{array}$ & $\begin{array}{r}0 \\
24\end{array}$ & $\begin{array}{r}0 \\
20\end{array}$ & $\begin{array}{r}0 \\
20\end{array}$ \\
\hline 10 & $\begin{array}{r}0 \\
20\end{array}$ & $\begin{array}{r}0 \\
20\end{array}$ & $\begin{array}{r}0 \\
20\end{array}$ & $\begin{array}{r}0 \\
20\end{array}$ & $\begin{array}{r}0 \\
24\end{array}$ & $\begin{array}{r}0 \\
24\end{array}$ & $\begin{array}{r}0 \\
20\end{array}$ & $\begin{array}{r}0 \\
20\end{array}$ \\
\hline 15 & $\begin{array}{r}0 \\
20\end{array}$ & $\begin{array}{r}0 \\
20\end{array}$ & $\begin{array}{r}0 \\
19\end{array}$ & $\begin{array}{r}0 \\
20\end{array}$ & $\begin{array}{r}0 \\
24\end{array}$ & $\begin{array}{r}0 \\
24\end{array}$ & $\begin{array}{r}3 \\
20\end{array}$ & $\begin{array}{r}1 \\
20\end{array}$ \\
\hline 19 & $\begin{array}{r}0 \\
20\end{array}$ & $\begin{array}{r}0 \\
20\end{array}$ & $\begin{array}{r}1 \\
19\end{array}$ & $\begin{array}{r}0 \\
20\end{array}$ & $\begin{array}{r}2 \\
24\end{array}$ & $\begin{array}{r}8 \\
24\end{array}$ & $\begin{array}{r}9 \\
12\end{array}$ & $\begin{array}{l}14 \\
18\end{array}$ \\
\hline 21 & $\begin{array}{r}0 \\
20\end{array}$ & $\begin{array}{r}0 \\
19\end{array}$ & $\begin{array}{r}4 \\
13\end{array}$ & $\begin{array}{l}11 \\
18\end{array}$ & $\begin{array}{r}6 \\
24\end{array}$ & $\begin{array}{l}18 \\
24\end{array}$ & $\begin{array}{l}12 \\
12\end{array}$ & $\begin{array}{l}16 \\
16\end{array}$ \\
\hline 27 & $\begin{array}{r}2 \\
40\end{array}$ & $\begin{array}{r}1 \\
40\end{array}$ & $\begin{array}{r}8 \\
11\end{array}$ & $\begin{array}{l}13 \\
13\end{array}$ & $\begin{array}{l}10 \\
20\end{array}$ & $\begin{array}{l}19 \\
19\end{array}$ & & \\
\hline 33 & $\begin{array}{l}10 \\
12\end{array}$ & $\begin{array}{r}7 \\
17\end{array}$ & $\begin{array}{l}7 \\
8\end{array}$ & $\begin{array}{l}10 \\
10\end{array}$ & $\begin{array}{l}14 \\
18\end{array}$ & $\begin{array}{l}16 \\
16\end{array}$ & & \\
\hline 43 & $\begin{array}{l}10 \\
10\end{array}$ & $\begin{array}{l}17 \\
17\end{array}$ & $\begin{array}{l}5 \\
5\end{array}$ & $\begin{array}{l}8 \\
8\end{array}$ & $\begin{array}{l}16 \\
16\end{array}$ & $\begin{array}{l}10 \\
10\end{array}$ & & \\
\hline
\end{tabular}

Le chiffre indique le nombre de crises audiogènes.

Le chiffre inférieur indique le nombre d'animaux utilisés.

\section{TABLEAU 4}

Crise audiogène et ration magnésique :

correction de la crise audiogène par le chlorure de magnésium

\begin{tabular}{cccc}
\hline Souche & OF1 & OF1 & $N$ \\
\hline Sexe & $\delta$ & $q$ & \\
\hline
\end{tabular}

Poids $\ldots \ldots \ldots \ldots \ldots \ldots . \quad 30,8 \pm 0,8 \quad 29,4 \pm 0,6$

\begin{tabular}{rlll}
\hline Durée de la carence $(\mathrm{j}) \ldots \ldots$ & 43 & 43 \\
\hline \multicolumn{5}{c}{ p. 100 de crises audiogènes } \\
$\begin{array}{c}\text { Chlorure de } \\
(14 \mathrm{mg} / \mathrm{j} / \mathrm{VB})\end{array} * \begin{array}{c}\text { magnésium } \\
\ldots \ldots \ldots\end{array}$ & 0 & 0 & $2 \times 30$ \\
\hline
\end{tabular}

Les chiffres indiquent le pourcentage de crises obtenues.

$\mathbf{N}=$ Nombre d'animaux utilisés pour chaque expérimentation.

* Pendant les 10 jours précédant le fest audiogène. 
TABLEAU 5

Profection contre la crise audiogène

\begin{tabular}{|c|c|c|c|}
\hline Souche & OF1 & OF1 & $N$ \\
\hline Sexe & $0^{*}$ & 우 & \\
\hline Poids (g) ... & $30,8( \pm 0,8)$ & $29,4( \pm 0,8)$ & \\
\hline \multirow{2}{*}{ Durée de la carence (j)....... } & 43 & 43 & \\
\hline & \multicolumn{2}{|c|}{ p. 100 de crises audiogènes } & \\
\hline Diazépam $(10 \mathrm{mg} / \mathrm{kg}) * \ldots \ldots$ & 0 & 0 & $2 \times 30$ \\
\hline Amobarbital $(50 \mathrm{mg} / \mathrm{kg}) * \ldots$ & 0 & 0 & $2 \times 30$ \\
\hline Phénobarbital $(50 \mathrm{mg} / \mathrm{kg}) * \ldots$ & 0 & 0 & $2 \times 30$ \\
\hline $\begin{array}{c}\text { Dipropylacétate de sodium } \\
(100 \mathrm{mg} / \mathrm{kg})^{*} \ldots \ldots \ldots \ldots\end{array}$ & 0 & 0 & $2 \times 30$ \\
\hline Méprobamate $(139 \mathrm{mg} / \mathrm{kg}){ }^{*} \ldots$ & 0 & 0 & $2 \times 30$ \\
\hline
\end{tabular}

Les chiffres indiquent le pourcentage de crises audiogènes obtenues.

$\mathrm{N}=$ Nombre d'animaux utilisés pour chaque expérimentation.

$*=$ Doses efficaces à 100 p. 100.

TABLEAU 6

Action de la parachlorophénylalanine (300 mg/kg, IP, 24 h avant le test audiogène)

\begin{tabular}{|c|c|c|c|c|}
\hline & OF1 & $\mathrm{C} 57 \mathrm{BL} / \mathrm{R}$ & $A K R / R$ & $\mathrm{DBA} / 2 / \mathrm{M}$ \\
\hline Sexe & $\hat{\jmath}$ & $\partial$ & $\hat{\sigma}$ & 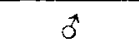 \\
\hline Durée de la carence & 43 & 43 & 43 & 21 \\
\hline$N \ldots \ldots \ldots \ldots \ldots \ldots \ldots \ldots$ & 30 & 30 & 30 & 30 \\
\hline PCPA $\mathrm{mg} / \mathrm{kg} \ldots \ldots \ldots \ldots \ldots$ & 300 & 300 & 300 & 300 \\
\hline Nombre de crises audiogènes. . & 0 & 0 & $\begin{array}{c}15 \\
(50 \text { p. } 100)\end{array}$ & $\begin{array}{c}18 \\
(60 \text { p. 100) }\end{array}$ \\
\hline
\end{tabular}

$N=$ Nombre de souris utilisées.

3. Substances aggravant la crise audiogène.

Pour toute cette expérimentation l'intensité sonore a été fixée à $80 \mathrm{~dB}$. Des essais préliminaires (tabl. 7) montrent que les souris non carencées, âgées de 70 jours, traitées par le sulfate d'amphétamine $\left(\mathrm{D}^{+}\right)$ou par la caféine ne font pas de crises audiogènes. 
TABLEAU 7

Action du sulfate d'amphétamine et de la caféine

Témoins non carencés

\begin{tabular}{|c|c|c|c|c|c|c|c|c|}
\hline \multirow{2}{*}{$\begin{array}{c}\text { Souche } \\
\text { Sexe }\end{array}$} & \multicolumn{2}{|c|}{ OF1 } & \multicolumn{2}{|c|}{$\mathrm{C} 57 \mathrm{BL} / \mathrm{R}$} & \multicolumn{2}{|c|}{ AKR/R } & \multicolumn{2}{|c|}{$\mathrm{DBA} / 2 / \mathrm{M}$} \\
\hline & o & 오 & 0 & q & 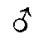 & $q$ & 3 & 古 \\
\hline Age (j) & 91 & 91 & 85 & 85 & 85 & 85 & 80 & 80 \\
\hline Amphétamine $(5 \mathrm{mg} / \mathrm{kg} \mathrm{IP}) \ldots$ & $\begin{array}{r}0 \\
20\end{array}$ & $\begin{array}{r}0 \\
20\end{array}$ & $\begin{array}{r}0 \\
20\end{array}$ & $\begin{array}{r}0 \\
20\end{array}$ & $\begin{array}{r}0 \\
20\end{array}$ & $\begin{array}{r}0 \\
20\end{array}$ & $\begin{array}{r}0 \\
20\end{array}$ & $\begin{array}{r}0 \\
20\end{array}$ \\
\hline Caféine $(25 \mathrm{mg} / \mathrm{kg} \mathrm{IP}) \ldots \ldots \ldots \ldots$ & $\begin{array}{r}0 \\
20\end{array}$ & $\begin{array}{r}0 \\
20\end{array}$ & $\begin{array}{r}0 \\
20\end{array}$ & $\begin{array}{r}0 \\
20\end{array}$ & $\begin{array}{r}0 \\
20\end{array}$ & $\begin{array}{r}0 \\
20\end{array}$ & $\begin{array}{r}0 \\
20\end{array}$ & $\begin{array}{r}0 \\
20\end{array}$ \\
\hline
\end{tabular}

Le chiffre supérieur indique le nombre de crises audiogènes.

Le chiffre inférieur indique le nombre d'animaux utilisés.

Sulfate d'amphéfamine $\left(\mathrm{D}^{+}\right)$. - Nous avons employé les 4 souches précédentes, mais en limitant la carence magnésique à 21 jours pour la souche OF1, à 15 jours pour les souches $C 57 B L / R$ et $A K R / R$ et à 10 jours pour les $D B A / 2 / M$. Une telle durée de carence est insuffisante à elle seule pour que l'on puisse observer des crises audiogènes. Le sulfate d'amphétamine $\left(\mathrm{D}^{+}\right)$a été dissous dans une solution aqueuse de $\mathrm{CINa}$ à $0,9 \mathrm{p} .100$ de manière à ce que chaque animal en reçoive $5 \mathrm{mg} / \mathrm{kg}$ sous un volume de $0,3 \mathrm{ml}$ (en intrapéritonéale $30 \mathrm{~min}$ avant la stimulation sonore).

Tous les animaux carencés, traités par le sulfate d'amphétamine $\left(D^{+}\right)$, ont fait une crise audiogène (tabl. 8).

TABLEAU 8

Action du sulfate diamphétamine et de la caféine. - Souris carencées en magnésium

\begin{tabular}{|c|c|c|c|c|c|c|c|c|}
\hline \multirow{2}{*}{$\begin{array}{c}\text { Souche } \\
\text { Sexe }\end{array}$} & \multicolumn{2}{|c|}{ OF1 } & \multicolumn{2}{|c|}{$\mathrm{C} 57 \mathrm{BL} / \mathrm{R}$} & \multicolumn{2}{|c|}{$A K R / R$} & \multicolumn{2}{|c|}{$\mathrm{DBA} / 2 / \mathrm{M}$} \\
\hline & $\hat{0}$ & q & $\hat{\sigma}$ & $q$ & $\hat{\sigma}$ & $q$ & $\hat{\sigma}$ & q \\
\hline Durée de carence (j) ............ & 21 & 21 & 15 & 15 & 15 & 15 & 10 & 10 \\
\hline Age (j) $\ldots \ldots \ldots \ldots \ldots \ldots \ldots \ldots$ & 91 & 91 & 85 & 85 & 85 & 85 & 80 & 80 \\
\hline Carencés témoins & $\begin{array}{r}0 \\
20\end{array}$ & $\begin{array}{r}0 \\
20\end{array}$ & $\begin{array}{r}0 \\
20\end{array}$ & $\begin{array}{r}0 \\
20\end{array}$ & $\begin{array}{r}0 \\
20\end{array}$ & $\begin{array}{r}0 \\
20\end{array}$ & $\begin{array}{r}0 \\
20\end{array}$ & $\begin{array}{r}0 \\
20\end{array}$ \\
\hline $\begin{array}{l}\text { Carencés }+ \text { amphétamine } \\
(5 \mathrm{mg} / \mathrm{kg} \text { IP }) \ldots \ldots \ldots \ldots\end{array}$ & $\begin{array}{l}20 \\
20\end{array}$ & $\begin{array}{l}20 \\
20\end{array}$ & $\begin{array}{l}20 \\
20\end{array}$ & $\begin{array}{l}20 \\
20\end{array}$ & $\begin{array}{l}20 \\
20\end{array}$ & $\begin{array}{l}20 \\
20\end{array}$ & $\begin{array}{l}20 \\
20\end{array}$ & $\begin{array}{l}20 \\
20\end{array}$ \\
\hline Carencés + caféine $(25 \mathrm{mg} / \mathrm{kg} I \mathrm{P})$ & $\begin{array}{l}20 \\
20\end{array}$ & $\begin{array}{l}20 \\
20\end{array}$ & $\begin{array}{l}20 \\
20\end{array}$ & $\begin{array}{l}20 \\
20\end{array}$ & $\begin{array}{l}20 \\
20\end{array}$ & $\begin{array}{l}20 \\
20\end{array}$ & $\begin{array}{l}20 \\
20\end{array}$ & $\begin{array}{l}20 \\
20\end{array}$ \\
\hline
\end{tabular}

Le chiffre supérieur indique le nombre de crises audiogènes.

Le chiffre inférieur indique le nombre d'animaux utilisés.

Caféine. - Les 4 souches ont été utilisées comme précédemment. La caféine ( $25 \mathrm{mg} / \mathrm{kg}$ par voie intrapéritonéale) a été injectée $30 \mathrm{~min}$ avant la stimulation sonore. 
Les témoins carencés ont reçu chacun $0,3 \mathrm{ml}$ de chlorure de sodium à 0,9 p. 100 . Tous les animaux carencés traités par la caféine ont fait une crise audiogène (tabl. 8).

Isoniazide. - Cet essai n'a été pratiqué que sur souris OF1. Deux lots ont été constitués (témoins carencés depuis 21 jours et témoins non carencés). Pour chaque lot on a distingué d'une part les mâles, d'autre part les femelles. Tous les animaux ont reçu de l'isoniazide en sous-cutanée à raison de $100 \mathrm{mg} / \mathrm{kg}$. Ils ont été soumis une heure après l'injection à une stimulation sonore dont l'intensité était de $80 \mathrm{~dB}$.

Pour les animaux témoins, non carencés, nous avons obtenu 43,3 p. 100 de crises audiogènes pour les mâles et 36,6 p. 100 pour les femelles. Les animaux carencés quel que soit leur sexe, ont tous fait une crise audiogène (tabl. 9).

TABLEAU 9

Action de l'isoniazide $(100 \mathrm{mg} / \mathrm{kg} \mathrm{SC})$

\begin{tabular}{|c|c|c|c|c|}
\hline \multirow{2}{*}{$\begin{array}{c}\text { Souche } \\
\text { Sexe }\end{array}$} & \multicolumn{2}{|c|}{$\begin{array}{c}\text { OF1 } \\
\text { (non carencées) }\end{array}$} & \multicolumn{2}{|c|}{$\begin{array}{c}\text { OF1 } \\
\text { (carencées pendant } 21 \text { jours) }\end{array}$} \\
\hline & $0^{x}$ & q & $\hat{\sigma}$ & p \\
\hline Age (j) $\ldots \ldots \ldots \ldots \ldots$ & 91 & 91 & 91 & 91 \\
\hline Poids $\ldots \ldots \ldots \ldots \ldots \ldots \ldots$ & $33,8 \pm 0,4$ & $28,6 \pm 0,2$ & $28,2 \pm 0,6$ & $26,2 \pm 0,2$ \\
\hline Nombre de souris ........ & 30 & 30 & 30 & 30 \\
\hline $\begin{array}{c}\text { Nombre de crisesaudiogè- } \\
\text { nes } \ldots \ldots \ldots \ldots \ldots \ldots \ldots\end{array}$ & $\frac{13}{(43,3 \text { p. } 100)}$ & $\begin{array}{c}11 \\
(36,6 \text { p. } 100)\end{array}$ & $\begin{array}{l}30 \\
(100 \text { p. } 100)\end{array}$ & $(100$ p. 100$)$ \\
\hline
\end{tabular}

Action des neuroleptiques (tabl. 10). - Nous n'avons utilisé que des souris de souche OF1, carencées en magnésium pendant 43 jours. L'intensité sonore était de $80 \mathrm{~dB}$ et la

TABLEAU 10

Action des neuroleptiques

(souche OF1 carencée depuis 43 jours)

\begin{tabular}{|c|c|c|c|c|c|c|}
\hline \multirow[b]{2}{*}{ Sexe } & \multicolumn{2}{|c|}{ p. 100 de crises } & \multicolumn{2}{|c|}{ p. 100 de mortalité } & \multicolumn{2}{|c|}{$N$} \\
\hline & $\widehat{\delta}$ & q & 0 & q & 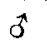 & $q$ \\
\hline Carencés témoins $\ldots \ldots \ldots \ldots \ldots \ldots$ & 100 & 100 & 20 & 10 & 60 & 60 \\
\hline $\begin{array}{l}\text { Carencés + chlorpromazine } \\
(4 \mathrm{mg} / \mathrm{kg} \text { IP) } \ldots \ldots \ldots \ldots \ldots \ldots \ldots \ldots\end{array}$ & 100 & 100 & 46,6 & 23,3 & 30 & 30 \\
\hline Carencés + réserpine $(1 \mathrm{mg} / \mathrm{kg}$ IP) $\ldots$ & 100 & 100 & 86,6 & 83,3 & 30 & 30 \\
\hline Carencés + halopéridol (1 $\mathrm{mg} / \mathrm{kg}$ IP) & 100 & 100 & 70 & 70 & 30 & 30 \\
\hline $\begin{array}{l}\text { Carencés + lévomépromazine } \\
(4 \mathrm{mg} / \mathrm{kg} \text { IP) } \ldots \ldots \ldots \ldots \ldots \ldots \ldots\end{array}$ & 100 & 100 & 30 & 10 & 30 & 30 \\
\hline
\end{tabular}

$\mathbf{N}=$ Nombre de souris utilisées. 
fréquence de $10 \mathrm{kHz}$. Les différents produits ont été administrés 30 min avant le test audiogène. On a observé que les neuroleptiques (chlorpromazine, réserpine, lévomépromazine, halopéridol) ne protègent pas les animaux carencés contre la crise audiogène ef même, aggravent la violence de la crise en augmentant le pourcentage de mortalité.

\section{Discussion.}

Au cours de cette éfude nous avons montré qu'en modifiant l'apport alimenlaire en magnésium on pouvait obtenir des crises audiogènes chez des animaux adultes, de souches non consanguines, peu sensibles à cette crise ef également chez des animaux qui avaient perdu avec l'âge leur sensibilité à la crise audiogène. Nous avons également montré que les crises audiogènes étaient liées à la carence magnésique puisque l'administration pendant 10 jours de chlorure de magnésium permettait de les supprimer. Nous pouvons donc proposer un classement (par ordre décroissant) de la sensibilité des différentes souches en fonction de la durée de la carence : $D B A / 2 / M$

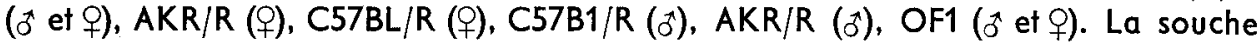
$\mathrm{DBA} / 2 / \mathrm{M}$ s'est montrée la plus sensible à la crise audiogène après une courte période de carence ; par contre la lignée OF1 n'a été sensible qu'après une période de carence plus prolongée. Nous avons constaté que dans la souche AKR/R et dans la souche $C 57 \mathrm{BL} / \mathrm{R}$ les femelles étaient plus sensibles que les mâles et ceci à partir du $21 \mathrm{e}$ jour de carence. Un tel résultat est à rapprocher de l'observation de Seelig ef al. (1975) pour l'espèce humaine où les formes neuromusculaires du déficit magnésique sont plus fréquentes chez la femme que chez l'homme.

L'intensité sonore utilisée au cours de ce travail pour sensibiliser l'animal carencé en magnésium était nettement moins imporłante que celle utilisée par plusieurs auteurs (Fuller ef al., 1950 ; Schlesinter et Boggan, 1968) pour l'éfude de la crise audiogène sur des lignées sensibles. La carence magnésique permet donc d'abaisser considérablement l'intensité sonore nécessaire au test audiogène. Ceci confirme pour la souris l'observation faite, pour le rat, par Chutkow (1971).

Mishra ef Perey (1960) avaient mis en évidence le risque d'infection au niveau de l'oreille interne chez le rat carencé en magnésium et avaient envisagé la possibilité de l'intervention d'une felle infection lors de l'apparition des crises audiogènes. Cela nous paraît peu probable chez la souris (Chance, 1963). Cependant, nous avons vérifié qu'une carence de 43 jours, pendant laquelle nous avons associé une couverture antibiotique afin d'empêcher une telle infection (ampicilline $50 \mathrm{mg} / \mathrm{kg}$ à partir du $33^{e}$ jour jusqu'au 43e jour) permettait de sensibiliser tous les animaux à la crise audiogène. Afin d'éliminer le risque d'une sensibilisation à la crise audiogène par cet antibiotique, nous avons vérifié que des animaux non carencés, traités dans les mêmes conditions, n'étaient pas sensibilisés à la crise audiogène.

L'étude neuropharmacologique de la crise audiogène chez l'animal « sensible » faite par plusieurs auteurs a permis de définir le rôle des neuromédiateurs. Lehmann et Busnel (1963), Jobé, Picchioni et Chin (1973a) ont montré qu'une déplétion cérébrale en sérotonine augmentait le nombre de crises. Pour Schlesinger et Boggan (1968), Jobé, Picchioni et Chin (1973b) il existerait une corrélation entre la sévérité de la crise et la baisse importante de la nor-épinéphrine. Par contre, aucune corrélation 
n'existerait entre la baisse de la dopamine cérébrale et la sévérité de la crise audiogène (Jobé, Picchioni et Chin, 1973b). On peut donc penser que la très grande sensibilité de l'animal carencé en magnésium serait due, d'une part à une hypersensibilité acoustique, d'autre part à une baisse importante de la sérotonine et de la nor-épinéphrine cérébrales. Le rôle de l'acide gamma aminobutyrique (GABA) a été envisagé dans cette étude. L'isoniazide convulsivant qui abaisse le GABA cérébral s'est révélé capable de sensibiliser à la crise audiogène tous les animaux carencés. Le fait qu'une seule injection de dipropylacétate de sodium, qui augmente le taux de GABA cérébral (Simler ef al., 1971 ; Wood et Abraham, 1971), permette de protéger de la crise les animaux carencés depuis 43 jours, nous conduit à penser que la carence magnésique, dans une certaine mesure, entraînerait un déficit en GABA cérébral.

L'action de la parachlorophénylalanine (PCPA) dans la crise audiogène chez l'animal « sensible » a été envisagée par plusieurs auteurs mais leurs conclusions sont contradictoires. Pour Jobé, Picchioni et Chin (1973a) chez le rat et pour Schlesinger (1970) chez la souris la PCPA augmente le nombre de crises audiogènes ainsi que la sévérité de ces crises. Alexandre et Kopeloff (1977) ont montré que la PCPA, chez la souris « sensible » et chez la souris carencée en magnésium (Alexandre et Kopeloff, 1978), permettait de protéger un certain nombre d'animaux de la crise audiogène. Cette étude confirme l'observation d'Alexander et Kopeloff (1978). La PCPA est capable de protéger totalement les souches OF1 et $C 57 \mathrm{BL} / \mathrm{R}$ et partiellement les souches $A K R / R$ et $D B A / 2 / M$. Toutefois aucun mécanisme d'action ne peut être proposé.

II est reconnu que l'amphétamine a des propriétés inhibitrices de la crise audiogène (Mercier, 1953 ; Lehmann, 1967), bien qu'elle entraîne une réduction du taux cérébral de nor-épinéphrine. Ces auteurs ont expliqué cela par une libération de nor-épinéphrine physiologiquement active et ensuite par sa destruction. Chez l'animal carencé en magnésium cette protection disparaît et, au contraire, l'amphétamine permet de sensibiliser tous les animaux à la crise audiogène après une courte période de carence (10,15 ou 21 jours). Ceci pourrait s'expliquer par une destruction totale de la norépinéphrine sans libération de nor-épinéphrine active et on peut penser que le magnésium joue un rôle dans cette libération.

La caféine, dont le mécanisme d'action est connu (baisse de la nor-épinéphrine ef de la sérotonine cérébrales. Berkowitz ef al., 1970 ; Corrodi ef al., 1972) permet de sensibiliser à la crise audiogène tous les animaux après une courte période de carence (10 à 21 jours selon la souche). On peut donc envisager l'hypothèse selon laquelle la caféine, chez l'animal carencé en magnésium, permettrait d'abaisser le taux cérébral de sérotonine et de nor-épinéphrine à un niveau tel que l'animal se trouve sensibilisé à la crise audiogène après une courte période de carence.

Nous avons envisagé l'action des neuroleptiques et nous avons montré qu'ils étaient incapables de protéger de la crise audiogène l'animal carencé en magnésium ef même qu'ils aggravaient la violence de cette crise en augmentant le pourcentage de mortalité. Pour la réserpine ce résultat était prévisible car cette substance entraîne une déplétion cérébrale en sérotonine (Lehmann, 1963). Parmi les neuroleptiques utilisés nous pouvons remarquer que plus le produit est catatonigène plus il aggrave la violence de la crise et que cette aggravation va de pair avec la multiplication des signes extrapyramidaux observés en clinique humaine. 


\section{Conclusion.}

Cette étude souligne l'importance du facteur alimentaire dans la sensibilité à la crise audiogène et la mauvaise folérance aux neuroleptiques de l'animal carencé en magnésium. Ce dernier point est à rapprocher d'une observation analogue en clinique humaine faite par Durlach (1979).

Reçu en décembre 1978.

Accepté en décembre 1980.

Remerciements. - Tous les animaux utilisés dans ce travail nous ont été gracieusement fournis par les laboratoires Meram que nous tenons à remercier.

\section{Références}

ALEXANDER G. J., KOPELOFF L. M., 1977. P-chlorophenylalanine mediated decrease in susceptibility to audiogenic seizures in imbred mice. Neuropharmacology, 16, 405-409.

ALEXANDER G. J., KOPELOFF L. M., 1978. Anticonvulsive effect of p-chlorophenylalanine in mice on a low magnesium diet. Pharmacol. Bioch. Behav., 8, 291-293.

BERKOWITZ B. A., TARVER J. H., SPECTOR S., 1970. Release of norepinephrine in the central nervous system by theophylline and caffeine. Eur. J. Pharmacol., 10, 64-71.

BOGGAN W. O., FREEDMANN D. X., LOVELL D. A., 1971. Studies in audiogenic susceptibility. Psychopharmacology, 20, 48-56.

BUCK D. R., MAHONEY A. W., HENDRICKS D. G., 1976. Effect of magnesium deficiency on non specific excitability level (NEL) and audiogenic seizure susceptibility. Pharmacol. Biochem. Behav., 5, 529-534.

BUCHEL L., DEBAY A., LEVY G., TANGUY O., 1961. Conditions de la sensibilisation des rongeurs par les hydrazides à la crise convulsive audiogène. Etude des substances protectrices. Thérapie, 16, $729-742$.

BUSNEL R. G., LEHMANN A., 1960. Nouvelles données pharmacodynamiques relatives à la crise audiogène de la souris. J. Physiol., 52, 37-38.

BUSNEL R. G., LEHMANN A., 1961. Action de convulsivants chimiques sur des souris de lignées sensibles et résisłantes à la crise audiogène. J. Physiol., 53, 285-286.

CHAUCHARD P., MAZOUE H., 1963. Effet comparé de divers excitants nerveux. J. Physiol., 55, 229230.

CHANCE M. R. A., 1963. A biological perspective on convulsions. Coll. int. CNRS, 112, 15-43. CNRS Ed.

CIELSIENSKY C., MAITRE M., SIMLER S., 1972. Mécanisme d'action d'un anti-convulsivant le n-dipropylacétate de sodium. J. Physiol., 61, 109a.

CORRODI H., FUXE K., JONSSON G., 1972. Effects of caffeine on central monoamine neurons. J. Pharm. Pharmacol., 24, 155-158.

CHUTKOW J. G., 1965. Studies on the metabolism of magnesium in the magnesium deficient rat. J. Lab. clin. Med., june, 918-926.

CHUTKOW J. G., 1971. Role of magnesium in neuromuscular physiology, 113-134. In DURLACH J., 1 er Symp. Déf. magn. path. hum., $1 \mathrm{er}$ vol. des rapports. Vittel, SGEMV éd.

DECKARD B., LIEFF B., SCHLESINGER H., DE FRIES J. C., 1976. Developmental patterns of seizure susceptibility in imbred strains of mice. Dev. Psychobiol., 9, 17-24.

DONALDSON H. H., 1924. The rat. Mem. Wistar Inst., 6, 2ed Phil.

DURLACH J., 1976. Neurological manifestations of magnesium imbalance. In Hand book of clinical neurology, Vol. 28, part 2, 545-579. Vinken, Bruyn ed.

DURLACH J., 1977. Aspects cliniques du déficit magnésique chronique. Vie méd., 6, 146-180. 
DURLACH J., 1979. 6e colloque français annuel sur le magnésium. Rev. fr. Endocr. clin. Nutr. Metab., 20, $171-178$.

FRINGS H. M., FULLER J. L., GINSBURG B. E., ROSS S., 1952. Standardization of nomenclature describing audiogenic seizures in mice. Behavior., 4, 157-160.

FULLER J. L., EASLER C., SMITH M., 1950. Inheritance of audiogenic seizure susceptibility in the mouse. Genetics, 35, 622-632.

FULLER J. L., SJURSEN F. H., 1967. Audiogenic seizures in cleven mouse strains. J. Hered., 58, 135140.

GINSBURG B. E., 1963. Causal mecanisms in audiogenic seizures. Coll. int. CNRS, 112, 227-240. CNRS Ed.

GRIFFITHS W. J., 1947. Audiogenic fits produced by magnesium deficiency in tame domestic norway rats and in wild norway and alexandrine rats. Amer. J. Physiol., 149, 135-141.

JOBE P. C., PICCHIONI A. L., CHIN L., 1973a. Role of brain 5-hydroxytryptamine in audiogenic seizure in the rat. Life. Sci., 13, 1-13.

JOBE P. C., PICCHIONI A. L., CHIN L., 1973b. Role of brain norepinephrine in audiogenic seizure in the rat. J. Pharmacol. exp. ther., 184, 1-10.

LEHMANN A., 1963. Action des hydrazides convulsivants sur l'épilepsie acoustique dite crise audiogène chez la souris. J. Physiol., 55, 282-283.

LEHMANN A., 1967. Audiogenic seizures data in mice supporting new theories of biogenic amines mecanisms in the central nervous system. Life Sci., 6, 1423-1431.

LEHMANN A., BUSNEL R. G., 1963. Le métabolisme de la sérotonine dans ses rapports avec la crise audiogène de la souris ef ses variations sous l'influence de divers composés psychotropes. Coll. int. CNRS, 112, 453-469. CNRS ed.

MERCIER J., 1953. Ełude physiologique ef pharmacodynamique des crises épileptoides expérimentales. Th. Doct. Sci., Marseille No 097843, 613 pp.

MISHRA R. K., PEREY F., 1960. Studies on experimental magnesium deficiency in the albinos rat. Rev. canod. Biol., 197, 143-153.

SEELING M. S., BERGER A. R., SPIELHOLZ N., 1975. Latent tetany anxiety, marginal magneisum deficit and normocalcemia. Dis. nerv. Syst., 36, 461-465.

SCHLESINGER K., 1970. Genetic of audiogenic seizure (3). Life Sci., 9, 721-729.

SCHLESINGER K., BOGGAN W., 1968. Genetic of audiogenic seizure (2). Life Sci., 7, 437-447.

SIMLER S., RANDRIANARISOA H., LEHMANN A., MANDEL P., 1968. Effets du di-n-propylacétate, sur les crises audiogènes de la souris. J. Physiol., 60, Suppl. 2, 547.

STOUDENSON B., 1924. In VASSILIEV Y. A. Sur la nature des réactions de Parfenov (Russ.). Fysiol. J. $6,80$.

SUTER C., KLINGMAN W. O., BOGGS D., MARCKS R. D.,1957. Sound-induced seizures in animals : magnesium deficiency and sound-induced seizures in rats. Neurol. (Mineap.), 8, 125-128.

SWINYARD E. A., 1963. Some physiological properties of audiogenic seizures in mice and their alteration by drugs. Coll. int. CNRS, 112, 405-421. CNRS Ed.

VOSKIAN H., 1970. Apports nouveaux à l'étude du magnésium en biologie. Thèse Doct. Pharm. (Etat), Paris, sér. E no 204, 208 pp.

WOOD J. D., ABRAHAM D. E., 1971. The comparative effects of various hydrazides on gammaaminobutyric acid and its metabolism. J. Neurochem., 18, 1017-1025. 